\title{
A Generalized Regular Form for Multivariable Sliding Mode Control
}

\author{
W. PERRUQUETTI*, J.P. RICHARD and P. BORNE \\ Laboratory of Automatic Control (LAIL UPRESA CNRS 8021), \\ Ecole Centrale de LILLE, BP 48, 59651 Villeneuve d'Ascq Cedex - France
}

(Received 18 July 2000)

The paper shows how to compute a diffeomorphic state space transformation in order to put the initial mutivariable nonlinear model into an appropriate regular form. This form is an extension of the one proposed by Lukyanov and Utkin [9], and constitutes a guidance for a "natural" choice of the sliding surface. Then stabilization is achieved via a sliding mode strategy. In order to overcome the chattering phenomenon, a new non linear gain is introduced.

Keywords: Sliding mode control; Multivariable systems; Non linear systems; Stabilization

\section{INTRODUCTION}

Sliding mode control is based on variable structure systems theory: the control commutates in order to force the systems motions to behave on a desired surface (called the sliding surface). Sliding regimes are unaffected by perturbations satisfying the well-known matching conditions (see $[1,2,12])$. The choice of the surface is mostly related to some stabilization problem: the shape of the surface is selected $a$ priori, leading to a set of parameters that are to be computed (adjusted) in order to obtain the desired dynamics $([2,3,10,15])$. For

*Corresponding author. e-mail: wilfrid.perruquetti@ec-lille.fr 
LTI systems, a natural shape for the sliding surface is a linear one (hyperplan), but for nonlinear ones, one can tranform the initial system into:

1. a linear one and then, select an hyperplan (see [4, 12-14]);

2. transform the initial system into an appropriate form which provides a guide for the choice of the surface.

The last approch is investigated in this paper. For this, we consider MIMO systems modeled by:

$$
\dot{x}=f(x)+G(x) u, \quad(M)
$$

where $x \in \mathbb{R}^{n}$ is the state vector, $u \in \mathbb{R}^{m}$ is the control vector ( $m$ inputs), $f: \mathbb{R}^{n} \rightarrow \mathbb{R}^{n}$, a smooth drift vector field, $G(x)=\left(g_{1}(x), \ldots, g_{m}(x)\right)$ is an $(n \times m)$-matrix and the $g_{i}: \mathbb{R}^{n} \rightarrow \mathbb{R}^{n}$ are smooth vector fields, $g_{i j}(x)$ is the control gain of the $j$ th input acting on the $i$ th state space variable.

Section 2 gives first a static feedback in order to obtain (1) with an input gain matrix of full rank and then, solves the question of obtaining (through a regular change of coordinates) an equivalent "regular form" to (1), defined as follows:

$$
(R F) \equiv\left\{\begin{array}{c}
\dot{z}_{1}=f_{1}^{R}\left(z_{1}, z_{2}\right)+G^{R}\left(z_{1}, z_{2}\right) u \\
\dot{z}_{2}=f_{2}^{R}\left(z_{1}, z_{2}\right), \\
z_{1} \in \mathbb{R}^{d}, z_{2} \in \mathbb{R}^{(n-d)}
\end{array}\right.
$$

Here, the number $d$ may be greater or smaller than the number of input $m$ (see Theorem 2), whereas in [9] $d=m$ : thus our result is more general. On the basis of such a regular form, results of Section 3 are devoted to the stabilizing controller design. Moreover, in order to overcome the "chattering" phenomenon, that is an important drawback of sliding mode control, we introduce a new nonlinear gain for the signum function such that the gain decreases as the motions converge towards the origin.

The following notations are used throughout the paper:

- for smooth $n$-vector fields, $f(x), g(x),[8]:[f, g](x) \triangleq(\partial g(x) / \partial x)$ $f(x)-(\partial f(x) / \partial x) g(x)$, (Lie bracket). The $A d$ operator is defined by $A d_{f}^{0} g(x) \triangleq g(x), A d_{f}^{1}(g)(x) \triangleq[f, g](x), \ldots, A d_{f}^{k} g(x) \triangleq\left[f, A d_{f}^{k-1} g\right](x)$.

- for a smooth real-valued function $\lambda(x),[8]: d \lambda(x) \triangleq\left(\left(\partial \lambda / \partial x_{1}\right), \ldots\right.$, $\left.\left(\partial \lambda / \partial x_{i}\right), \ldots,\left(\partial \lambda / \partial x_{n}\right)\right)$, (the "gradient" of $\left.\lambda\right)$. 
- $\operatorname{Sgn}(\zeta)$ and $\operatorname{Sgn}(z)$ are respectively scalar and vector signum functions defined as follows:

$$
\begin{gathered}
\operatorname{Sgn}(\zeta)=\left\{\begin{aligned}
-1 & \text { if } \zeta<0, \\
1 & \text { if } \zeta>0,
\end{aligned}\right. \\
\operatorname{SGN}(z)=\left(\operatorname{Sgn}\left(z_{1}\right), \ldots, \operatorname{Sgn}\left(z_{l}\right)\right), z \in \mathbb{R}^{l} .
\end{gathered}
$$

- $k(\cdot)$ stands for a function $k$ of the state variables that may be constant.

\section{OBTAINING THE REGULAR FORM}

The problem is to find a diffeomorphic state space transformation $z=\phi(x)$ changing $(M)(1)$ into $(R F)(2)$. The proposed procedure is: firstly, transform $(M)(1)$ into a model with full rank gain matrix; lastly, define conditions on the existence of a regular form.

\section{II.A. On the Rank of the Input Gain Matrix}

In [9], the hypothesis $\operatorname{rank}(G(x))=m$ was combined with some additional integrability conditions to obtain $(R F)(2)$ with $d=m$. The next theorem shows how to recover this hypothesis from the general case through a static feedback:

THEOREM 1 Let $x_{0} \in \mathbb{R}$. If $\operatorname{rank}\left(G\left(x_{0}\right)\right)=r$, then there is a static feedback $u=W(x)\left(v^{T}, 0, \ldots, 0\right)^{T}, v \in \mathbb{R}^{r}$, with $W$ nonsingular in a neighbourhood $\mathcal{N}\left(x_{0}\right)$ of $x_{0}$, such that:

$$
G(x) W(x)=\left(G^{\prime}(x) \mid 0_{n \times(m-r)}\right), \quad \forall x \in \mathcal{N}\left(x_{0}\right) .
$$

combined with $(M)$ (1) leads to:

$$
\dot{x}=f(x)+G^{\prime}(x) v, \quad\left(M^{\prime}\right)
$$

where $v$ is the new control vector, $G^{\prime}(x)$ is a $(n \times r)$-matrix of full rank $r$. Proof This comes out directly by performing a Gauss reduction on columns (using elementary columns transformations: interchange, sum, scalar multiplication by $a(x)$ ). Recall that in order to compute 
(5), one can perform on $G(x)$ and on the $(m \times m)$-identity matrix the same operation which leads respectively to the right-hand side of Eq. (5) and to $W(x)$. Note that $\operatorname{det}\left(W\left(x_{0}\right)\right)=1$.

In the following, we consider that $\operatorname{rank}\left(G\left(x_{0}\right)\right)=m$, otherwise $\left(\operatorname{rank}\left(G\left(x_{0}\right)\right)=r<m\right)$, the previous theorem allows us to consider the system $(M)(1)$ with static feedback (5) which leads to $\left(M^{\prime}\right)(6)$.

\section{II.B. On the Existence of a Regular Form}

The given results are local, but when asumption $\mathrm{H} 1$ (see theorem statements) holds everywhere in the state space, then the diffeomorphism is global and so are the results. The following statement can be regarded as an extension of previous results for nonlinear systems using differential geometric approach (see [8]):

THEOREM 2 Let $\Delta$ be a distribution such that:

(H1) $\Delta$ is nonsingular at $x_{0}$ (i.e., of constant dimension $\operatorname{dim} \Delta=d_{\Delta} \leq n$ ), (H2) $\Delta$ is involutive: $\forall \tau_{1} \in \Delta, \forall \tau_{2} \in \Delta:\left[\tau_{1}, \tau_{2}\right] \in \Delta$,

(H3) $\operatorname{span}\left\{g_{1}(x), \ldots, g_{m}(x)\right\} \subset \Delta$,

then there exist a neighbourhood $\mathcal{N}\left(x_{0}\right)$ of $x_{0}$ and a local diffeomorphism $z=\phi(x)$ defined on $\mathcal{N}\left(x_{0}\right)$, such that $(M)$ (1) is transformed into $(R F)$ (2) with $d=d_{\Delta} \leq n$.

The involutive closure ${ }^{1}$ of $\operatorname{span}\left\{g_{1}(x), \ldots, g_{m}(x)\right\}$ denoted by $\boldsymbol{\Delta}_{\mathbf{G}}$, satisfies the assumptions of Theorem 2.

Proof Under assumptions $(\mathrm{H} 1)-(\mathrm{H} 3)$, one can find $\left(n-d_{\Delta}\right)$ real valued functions $\lambda_{i}$ such that the anihilator $\Delta^{\perp}=\left\{\omega^{*} \in\left(\mathbb{R}^{n}\right)^{*}:\left\langle\omega^{*}\right.\right.$, $v\rangle=0, v \in \Delta\rangle$ of $\Delta$ is spanned by the covectors $d \lambda_{i}$ (Frobenius Theorem). Selecting $\phi_{d_{\Delta}+i} \triangleq \lambda_{i}$, for $i \in\left\{1 \cdots n-d_{\Delta}\right\}$ and completing the basis with real valued functions $\phi_{i}$, for $i \in\left\{1 \cdots d_{\Delta}\right\}$, such that $\operatorname{rank}\left(d \phi_{i}: i \in\{1 \cdots n\}\right)=n$, leads to the result.

Remark 1 Note that, for $m=1$ with $g_{1}\left(x_{0}\right) \neq 0$, the distribution $\Delta=\operatorname{span}\left\{g_{1}(x)\right\}$ is involutive and $(M)(1)$ can be transformed into $(R F)$ (2) with $d=\operatorname{dim} \Delta=1$. Now, if $\Delta=\operatorname{span}\left\{g_{1}(x), \ldots, g_{m}(x)\right\}$ satisfy

\footnotetext{
${ }^{1}$ see [8] for its construction.
} 
(H2) and (H3) of Theorem 2, then one obtains the classical result of [9] $\left(d=d_{\Delta}=m\right)$. So, our result is an extension which, as we will see in the following sections, provides a guidance for the design of sliding mode controller in the general case: $d$ may be greater or smaller ${ }^{2}$ than $m$.

\section{STABILIZING SLIDING MODE CONTROLLER}

From $(R F)(2)$, it is clear that, in order to make the origin of $(M)$ (1) stable, one can design a sliding mode control in the following way:

1. let $s=z_{1}-p\left(z_{2}\right) \in \mathbb{R}^{d}$, where the $d$-vector valued function $p$ is to be defined,

2. design a sliding mode control such that a sliding regime occurs on the manifold $s=0$ of dimension $(n-d)$,

3. define $p$ such that the origin $z_{2}=0$ is locally asymptotically stable for $\dot{z}_{2}=f_{2}^{R}\left(p\left(z_{2}\right), z_{2}\right)$ (dynamics in sliding regime).

In this procedure like in the center manifold theory (see [5]), there are two time scales: a fast one (the hitting phase is in finite time) and a slow one (asymptotic stabilization in sliding regime). Thus the sliding manifold plays the same role as the center manifold does in the theory of the same name.

This idea was first used in [9] under three assumptions: first $d=m$, secondly $\operatorname{rank}(G(x))=m$ and under an integrability condition on $G(x)$ (which is always fulfilled when $m=1$ ). In the following we give a generalization of these results. According to the previous section, we can distinguish the two following cases $^{3}$ :

1. $r=\operatorname{rank}\left(G\left(x_{0}\right)\right)=\operatorname{dim} \Delta_{G}$;

2. $r=\operatorname{rank}\left(G\left(x_{0}\right)\right)<\operatorname{dim} \Delta_{G}$.

Here, we shall consider the first Case 1; the second case will be treated through Example 2. Note that this first case is also considered in [9], but in the paper, all trajectories (this means for all initial

\footnotetext{
${ }^{2}$ if we do not use Theorem 1 .

${ }^{3}$ After eventually applying static feedback of Theorem 1 (see Section II.A).
} 
conditions) belonging to the surface must converge asymptotically. In our case, only local asymptotic stability is required. The sliding gain is calculated in such a way that the motions reach the sliding surface in its stable part.

THEOREM 3 Let us suppose that:

(H1) $\Delta_{G}$ exists with $\operatorname{dim} \Delta_{G}=r=\operatorname{rank}\left(G\left(x_{0}\right)\right)$, one obtains $(R F)$ (2) with $d=r$,

(H2) $f_{2}^{R}$ is at least $C^{1}\left(\mathbb{R}^{n} ; \mathbb{R}^{(n-r)}\right)$,

(H3) the origin $0 \in \mathbb{R}^{(n-r)}$ of system:

$$
\dot{z}_{2}=f_{2}^{R}\left(p\left(z_{2}\right), z_{2}\right),
$$

is locally asymptotically stable, with $p\left(z_{2}\right) \in C^{1}\left(\mathbb{R}^{(n-r)} ; \mathbb{R}^{\prime}\right), \quad p(0)=$ $0 \in \mathbb{R}^{r}$,

Then, defining the sliding surface as $s=z_{1}-p\left(z_{2}\right)$, we have:

(C1) there exists a gain $k(\cdot)$ providing a local asymptotic stabilization of the origin w.r.t. $(R F)(2)$, by means of the control:

$$
u=\left(G^{R}(z)\right)^{-1}\left(-f_{1}^{R}(z)-k(\cdot) S G N(s)+\frac{\partial p\left(z_{2}\right)}{\partial z_{2}} f_{2}^{R}(z)\right),
$$

(C2) if in addition $0 \in \mathbb{R}^{(n-r)}$, is globally asymptotically stable for (7), then the origin of $(R F)(2)$ is globally asymptotically stable under the control (8) defined with any non zero constant gain $k(\cdot)$.

Proof (constructive) (H1) implies $G^{R}(z)$ is nonsingular and $\left(G^{R}(z)\right)^{-1}$ exists (for all $z$ in a neighbourhood of $z_{0}$ ).

Point (C1): $\|x\|_{1}=\sum_{i=1}^{n}\left|x_{i}\right|, x \in \mathbb{R}^{n}$. From (H3) using a converse Lyapunov theorem (see [7]), there exist a Lyapunov function $V_{2}\left(z_{2}\right) \in C^{1}\left(\mathbb{R}^{(n-r)} ; \mathbb{R}_{+}\right)$and $\rho_{2}>0$ such that for every motion of system (7) starting in $S_{2}\left(\rho_{2}\right)=\left\{z_{2} \in \mathbb{R}^{(n-m)}: V_{2}\left(z_{2}\right) \leq \rho_{2}\right\}$, we have $\left.\dot{V}_{2}\right|_{(7)}<0$. So, let us consider $V(z)=(\alpha / 2) s^{\top} s+V_{2}\left(z_{2}\right)$, with $\alpha>0$, and restrict our attention to $S\left(\rho_{2}\right)=\left\{z \in \mathbb{R}^{n}: V(z) \leq \rho_{2}\right\}: \forall \rho_{2}<\infty, S\left(\rho_{2}\right)$ is compact.

$$
\left.\dot{V}\right|_{(2)}=\alpha s^{\top} \dot{s}+\frac{\partial V_{2}^{\top}}{\partial z_{2}}\left[f_{2}^{R}\left(z_{1}, z_{2}\right)-f_{2}^{R}\left(p\left(z_{2}\right), z_{2}\right)+f_{2}^{R}\left(p\left(z_{2}\right), z_{2}\right)\right] .
$$




$$
\begin{aligned}
\mid \frac{\partial V_{2}^{\top}}{\partial z_{2}} & {\left[f_{2}^{R}\left(z_{1}, z_{2}\right)-f_{2}^{R}\left(p\left(z_{2}\right), z_{2}\right)\right] \mid } \\
& \leq \sum_{i=1}^{n}\left|\left(\frac{\partial V_{2}}{\partial z_{2}}\right)_{i}\right|\left|\left(f_{2}^{R}\left(z_{1}, z_{2}\right)-f_{2}^{R}\left(p\left(z_{2}\right), z_{2}\right)\right)_{i}\right| \\
& \leq\left\|\frac{\partial V_{2}}{\partial z_{2}}\right\|_{1}\left\|f_{2}^{R}\left(z_{1}, z_{2}\right)-f_{2}^{R}\left(p\left(z_{2}\right), z_{2}\right)\right\|_{1}
\end{aligned}
$$

(H2) implies that $f_{2}^{R}$ is uniformly Lipschitz on $S\left(\rho_{2}\right)$, and as $\quad\left(z_{1}, z_{2}\right) \in S\left(\rho_{2}\right) \Rightarrow\left(p\left(z_{2}\right), z_{2}\right) \in S\left(\rho_{2}\right)$, thus $\exists L_{\rho_{2}}: \forall\left(z_{1}, z_{2}\right) \in S\left(\rho_{2}\right)$, $\left\|f_{2}^{R}\left(z_{1}, z_{2}\right)-f_{2}^{R}\left(p\left(z_{2}\right), z_{2}\right)\right\|_{1} \leq L_{\rho_{2}}\left\|z_{1}-p\left(z_{2}\right)\right\|_{1}=L_{\rho_{2}} s^{\top} \operatorname{SGN}(s)$. With control (8), this leads to:

$$
\begin{aligned}
\left.\dot{V}\right|_{(2)} \leq & -\alpha k(\cdot) s^{\top} \operatorname{SGN}(s)+L_{\rho_{2}}\left\|\frac{\partial V_{2}}{\partial z_{2}}\right\|_{1} s^{\top} \operatorname{SGN}(s) \\
& +\frac{\partial V_{2}^{\top}}{\partial z_{2}} f_{2}^{R}\left(p\left(z_{2}\right), z_{2}\right), \forall z \in S\left(\rho_{2}\right)
\end{aligned}
$$

We can now choose $k(\cdot)=1+\left(L_{\rho_{2}} / \alpha\right)\left\|\left(\partial V_{2} / \partial z_{2}\right)\right\|_{1}:\left.\dot{V}\right|_{(2)} \leq-\alpha s^{\top}$ $\operatorname{Sgn}(s)+\left(\partial V_{2} / \partial z_{2}\right)^{\top} f_{2}^{R}\left(p\left(z_{2}\right), z_{2}\right) \cdot V_{2}$ is a Lyapunov function for (7): if $z \in S\left(\rho_{2}\right) \subset \mathbb{R}^{n}$ then $z_{2} \in S_{2}\left(\rho_{2}\right) \subset \mathbb{R}^{(n-m)}$, so $\left.\dot{V}_{2}\right|_{(7)}=\left(\partial V_{2} / \partial z_{2} f_{2}^{R}\left(p\left(z_{2}\right)\right.\right.$, $\left.z_{2}\right)$ is negative define $\forall z_{2} \in S_{2}\left(\rho_{2}\right)$. Therefore, $\left(\partial V_{2} / \partial z_{2}\right)^{\top} f_{2}^{R}\left(p\left(z_{2}\right), z_{2}\right)$ is negative $\forall_{z} \in S\left(\rho_{2}\right) \subset \mathbb{R}^{n}$, which ends the proof.

Point (C2): first show that solution tends to the sliding surface in finite time (see for example [10]), then the result follows obviously.

THEOREM 4 If $(H 1)$ and $(H 2)$ of Theorem 3 hold and (H3) is replaced by: $\left(\mathrm{H3}^{\prime}\right)$ there exist a Lyapunov function $V_{2}\left(z_{2}\right)$ and a constant $\rho_{2}$ such that $S_{2}\left(\rho_{2}\right)=\left\{z_{2} \in \mathbb{R}^{(n-r)}: V_{2}\left(z_{2}\right) \leq \rho_{2}\right\}$ is an estimate of the domain of asymptotic stability of the origin $0_{2} \in \mathbb{R}^{(n-r)}$ of (7), then, the control $u$ defined by (8) with gain $k(\cdot)=k^{\prime}(\cdot)+\left(L_{\rho_{2}} / \alpha\right)\left\|\left(\partial V_{2} / \partial z_{2}\right)\right\|_{1}, k^{\prime}(z)>0$, $\alpha>0$, achieves asymptotic stability of the origin for system $(R F)(2)$ with $S\left(\rho_{2}\right)=\left\{z \in \mathbb{R}^{n}:(\alpha / 2) s^{\top} s+V_{2}\left(z_{2}\right) \leq \rho_{2}\right\}$ as an estimate of the domain of asymptotic stability.

Proof direct extension of the proof of Theorem 3.

Remark 2 If, in addition, $\lim _{z_{2} \rightarrow 0}\left\|\left(\partial V_{2} / \partial z_{2}\right)\right\|=\lim _{z \rightarrow 0} k^{\prime}(z)=0$, then "chattering" tends to zero as the motion approaches the origin. This condition is not very restrictive because, it is fulfilled if the 
Lyapunov function $V_{2}$ is, locally, at least quadratic $\left(k^{\prime}(\cdot)\right.$ can be set to $\left(L_{\rho_{2}} / \alpha\right)\left(\partial V_{2} / \partial z_{2}\right) \|_{1}$ for example). Note that $k^{\prime}(\cdot)$ can also be set to any other sigmoïd function zeroing at the origin: in [14], the sign function is replaced by a saturation function in order to smooth the discontinuity.

Example 1 Consider the following nonlinear system

$$
\begin{aligned}
& \dot{x}_{1}=x_{2}+u_{1}, \\
& \dot{x}_{2}=x_{1}+u_{1}+u_{2}, \\
& \dot{x}_{3}=x_{1} x_{3}+u_{1} .
\end{aligned}
$$

Distribution $\Delta=\operatorname{span}\left\{g_{1}, g_{2}\right\}, g_{1}=(1,1,1)^{\top}, g_{2}=(0,1,0)^{\top}$, is a twodimensional involutive distribution. In order to find $\phi$, one has to find a basis of $\Delta^{\perp}$ :

$$
\left\{\begin{array} { l } 
{ d \lambda g _ { 1 } = 0 } \\
{ d \lambda g _ { 2 } = 0 }
\end{array} \Longleftrightarrow \left\{\begin{array}{c}
\left(\partial \lambda / \partial x_{1}\right)+\left(\partial \lambda / \partial x_{2}\right)+\left(\partial \lambda / \partial x_{3}\right)=0 \\
\left(\partial \lambda / \partial x_{2}\right)=0
\end{array},\right.\right.
$$

which has solution $\lambda(x)=x_{1}-x_{3}$. Thus $z=\phi(x)=\left(x_{1}, x_{2}, x_{1}-x_{3}\right)^{\top}$ leads to (Theorem 2)

$$
\begin{aligned}
& \dot{z}_{1}=z_{2}+u_{1}, \\
& \dot{z}_{2}=z_{1}+u_{1}+u_{2}, \\
& \dot{z}_{3}=z_{2}-z_{1}\left(z_{1}-z_{3}\right) .
\end{aligned}
$$

Here $\operatorname{rank}(G)=2$, so according to Theorem 4 , let $u$ be defined as $u=\left(-x_{2}+v_{1}, x_{2}-x_{1}-v_{1}+v_{2}\right)^{\top}$, and let $s$ be defined as $s=\left(z_{1}-z_{3}\right.$, $\left.z_{2}+z_{3}\right)^{\top}=\left(x_{3}, x_{1}+x_{2}-x_{3}\right)^{\top}$. Let $\quad v_{1}=-\operatorname{Sgn}\left(x_{3}\right)+x_{2}-x_{1} x_{3}, \quad v_{2}=$ $-\operatorname{Sgn}\left(x_{1}+x_{2}-x_{3}\right)-x_{2}+x_{1} x_{3}$. Then, in sliding regime $\dot{z}_{3}=-z_{3}$, and global asymptotic stability of the origin is achieved (Fig. 1).

In order to reduce the chattering phenomenon one can use a nonlinear gain as proposed in Theorem $4: V_{2}\left(z_{2}\right)=\left(z_{2}^{2} / 2\right)$ leads to choose the gains of $\operatorname{Sgn}\left(x_{3}\right)$ as $\left|x_{1}-x_{3}\right|$ and of $\operatorname{Sgn}\left(x_{1}+x_{2}-x_{3}\right)$ as $2\left|x_{1}\right|$ $\left|x_{1}-x_{3}\right|$ (Fig. 2). Here, one can take two different gains, for this, rewrite $k(\cdot) s^{\top} \mathrm{SGN}(s)$ as $\sum_{i=1}^{r} k_{i}(\cdot)\left|s_{i}\right|$ in the proof of Theorem 3.

Example 2 This second example illustrates the case $\operatorname{rank}\left(G\left(x_{0}\right)\right)=$ $r<d=\operatorname{dim} \Delta_{G}$. The obtained regular form in Section I provides informations: as $\operatorname{rank}\left(G\left(x_{0}\right)\right)=r<d=\operatorname{dim} \Delta_{G}, G^{R}(z)$ the obtained input gain matrix in $(R F)(2)$ can be splitted into $T(z)$ a low triangular $(r \times r)$-matrix and $R(z)$ a rectangular matrix. This is possible due to the 

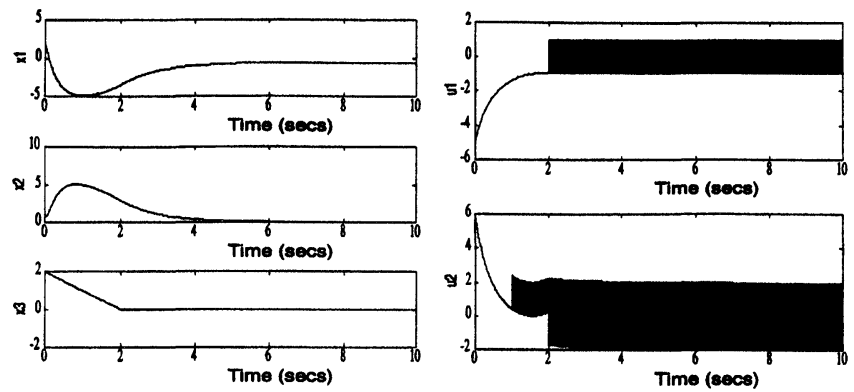

FIGURE 1 Stabilization of (10) using constant gains for sign functions.
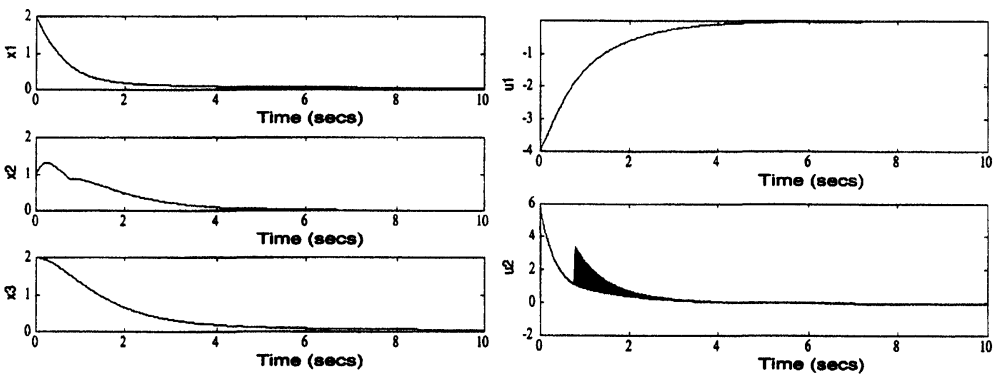

FIGURE 2 Stabilization of (10) using non linear gains obtained via Theorem 4.

use of the prestatic feedback $u=W(x)\left(v^{T}, 0, \ldots, 0\right)^{T}$ given by the Gauss reduction in the proof of Theorem 1 and using, if required, a permutation of the coordinates. Let us denote by $i \leq r$ the number of rows of $T$ which are necessary to span the rows of $R$. Then using $i$ integrators, one can obtained a dynamical extension for which Theorem 3 or 4 may apply. This procedure is going to be applied on a monocycle (see Fig. 3 ) with the pedaling rolling action $\left(u_{1}\right)$ and the rotating action $\left((d \theta / d t)=u_{2}\right)$.

The model, which also express the dynamics of a two-wheel cart (see [6]), is:

$$
\begin{aligned}
& \frac{d x_{1}}{d t}=\sin \theta u_{1}, \\
& \frac{d x_{2}}{d t}=\cos \theta u_{1}, \\
& \frac{d \theta}{d t}=u_{2} .
\end{aligned}
$$




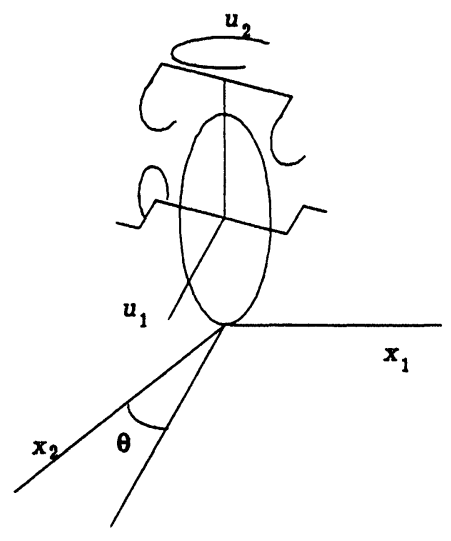

FIGURE 3 Monocycle.

Let $g_{1}(x)=(\sin \theta, \cos \theta, 0)^{\top}, g_{2}=(0,0,1)^{\top}$. As $g_{3}(x)=\left[g_{1}, \mathrm{~g}_{2}\right]=$ $(-\cos \theta, \sin \theta, 0)^{\top} \notin \operatorname{span}\left\{g_{1}(x), g_{2}(x)\right\}$. Let $\Delta_{G}=\operatorname{span}\left\{g_{1}(x), g_{2}(x)\right.$, $\left.g_{3}(x)\right\}$, this is a three-dimensional involutive distribution (compute the Lie brackets $\left[g_{1}, g_{3}\right]$ and $\left.\left[g_{2}, g_{3}\right]\right)$. Note that $\operatorname{dim}\left(\Delta_{G}\right)=3$ implies that (12) is locally accessible. The proof of Theorem 2 implies that for this system one cannot obtain $(R F)(2)$ with $d$ less than $3:$ thus (12) is in a regular form with $\operatorname{rank}\left(G\left(x_{0}\right)\right)=2<3=\operatorname{dim} \Delta_{G}$. From this regular form and the introduction of this example, permutting $x_{2}$ and $\theta$, it comes out that the last and first rows of $G^{R}(x)$ are dependent thus adding an integrator on the first input will break this link and leads to:

$$
\begin{aligned}
& \frac{d x_{1}}{d t}=\sin (\theta) \xi, \\
& \frac{d x_{2}}{d t}=\cos (\theta) \xi, \\
& \frac{d \theta}{d t}=u_{2}=v_{2}, \\
& \frac{d \xi}{d t}=v_{1}, \quad u_{1}=\xi .
\end{aligned}
$$

Then, according to Theorem 4, let $s=(\xi, \theta)^{\top}-p\left(x_{1}, x_{2}\right)$, thus $\dot{s}_{1}=v_{1}-\sin (\theta) \xi=-k_{1}(\cdot) \operatorname{Sgn}\left(s_{1}\right), \dot{s}_{2}=v_{2}-\cos (\theta) \xi=-k_{2}(\cdot) \operatorname{Sgn}\left(s_{2}\right)$. 
In sliding regime,

$$
\begin{aligned}
& \dot{x}_{1}=\sin \left(p_{2}\left(x_{1}, x_{2}\right)\right) p_{1}\left(x_{1}, x_{2}\right), \\
& \dot{x}_{2}=\cos \left(p_{2}\left(x_{1}, x_{2}\right)\right) p_{1}\left(x_{1}, x_{2}\right),
\end{aligned}
$$

which can be set respectively to the values $-\alpha x_{1}$ and $-\beta x_{2}$, for some $\alpha>0$ and $\beta>0$. For this, let $p_{1}\left(x_{1}, x_{2}\right)=-\left(\alpha x_{1} / \sin \left(\arctan \left(\alpha x_{1} / \beta x_{2}\right)\right)\right)$, and $p_{2}\left(x_{1}, x_{2}\right)=\arctan \left(\alpha x_{1} / \beta x_{2}\right)$. Moreover, in order to stabilize the origin of (12), we need $\theta$ to tend to zero which is achieved if $\lim _{t \rightarrow+\infty}\left(\arctan \left(\alpha x_{1}(t) / \beta x_{2}(t)\right)\right)=0$. A sufficient condition is $(-\alpha+\beta)<0$ (see [6]). Let us select $\alpha=2$ and $\beta=1$. Thus, global asymptotic stability of the origin of (12) is achieved using the control laws defined by:

$$
\begin{gathered}
u_{1}(t)=\int_{0}^{t}\left(-\operatorname{Sgn}\left(s_{1}\right)-\frac{\xi\left(4 \sin (\theta) x_{1}+\cos (\theta) x_{2}\right)}{x_{2} \sqrt{\left(1+4\left(x_{1}^{2} / x_{2}^{2}\right)\right)}}\right)(\tau) d \tau, \\
u_{2}=v_{2}=-k_{2}(\cdot) \operatorname{Sgn}\left(s_{2}\right)+\frac{2 \xi\left(\sin (\theta) x_{2}-\cos (\theta) x_{1}\right)}{4 x_{1}^{2}+x_{2}^{2}} \\
s_{1}=\xi+\frac{2 x_{1}}{\sin \left(\arctan \left(2 x_{1} / x_{2}\right)\right)}, \\
s_{2}=\theta-\arctan \left(\frac{2 x_{1}}{x_{2}}\right)
\end{gathered}
$$

Note that Gulden and Utkin ([6]) used an other approach, imposing the cart (or monocycle) to approach the origin according to a "Lyapunov Navigation Function" (the tracked path is derived from this Lyapunov function).

Figure 4 illustrates the stabilization of the origin under the controls (15) with $k_{i=1,2}(\cdot)=1$. The simulations were achieved from the following initial conditions: $x_{1}(0)=1.2, x_{2}(0)=2, \theta(0)=0.2(\mathrm{rd})$, $\xi(0)=0$ (no control at time zero). One can note that the first control $u_{1}$ is rather smooth (no chattering) : this is due to the presence of an integrator before the physical actuator. For the second control $u_{2}$, there is some chattering which can be smoothed using different technics (see for example [14] or sigmoïd functions). But, we can use a nonlinear gain as proposed in Theorem 4. Using the Lyapunov function $V_{2}\left(z_{2}\right)=x_{1}^{2}+\left(x_{2}^{2} / 2\right)$ leads to choose $k_{2}(\cdot)=0.2 \sqrt{4 x_{1}^{2}+x_{2}^{2}}$. 

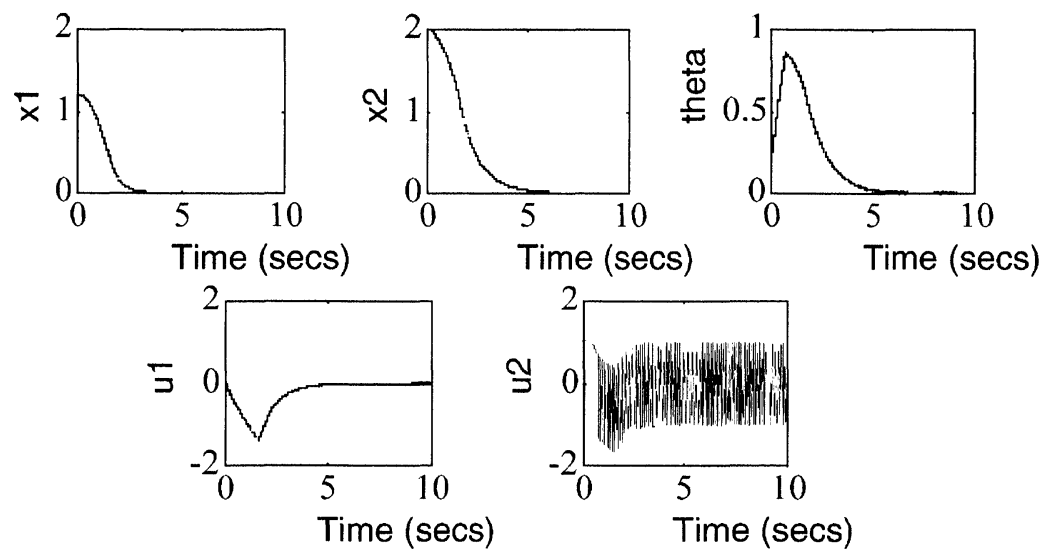

FIGURE 4 Stabilization of (12) using (14) with $k_{i=1,2}(\cdot)=1$.
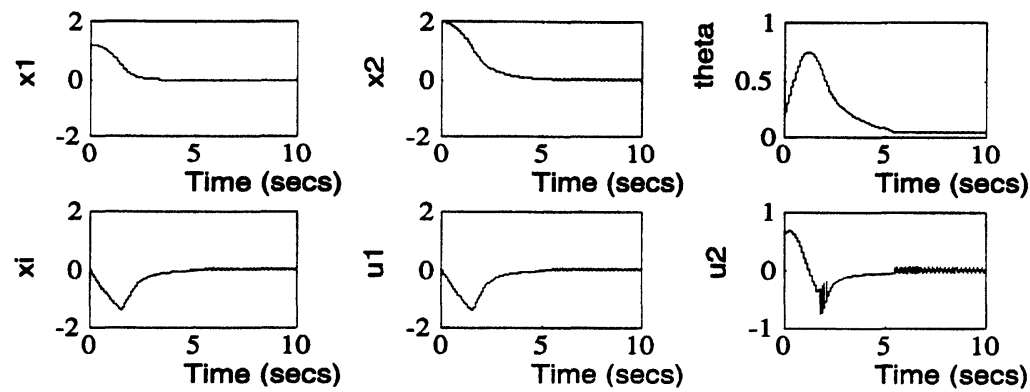

FIGURE 5 Stabilization of (12) using (14) with $k(\cdot)=0.2 \sqrt{4 x_{1}^{2}+x_{2}^{2}}$.

This gain replaces the gain " 1 " of the signum function $\operatorname{Sgn}\left(s_{2}\right)$ in (15) and yields the simulations of Figures 5 .

\section{CONCLUSION}

A first contribution of this paper is to complete the general problem of regular form initiated by Lukyanov and Utkin [9]:

1. The given results (Theorem 2 and consequences) are general ( $d$ may be greater or smaller than the number of inputs $m$ ) and do not depend on the rank of the input gain matrix (contrary to [9]). 
2. The construction of $\Delta_{G}$ is easily obtained applying the algorithm for the involutive closure of span $\left\{g_{1}(x), \ldots, g_{m}(\mathrm{x})\right\}$.

In addition, Section III gives the design of a sliding mode controller achieving asymptotic stabilization of the origin. The proposed design procedures take into account the rank of the input gain matrix and the dimension of $\Delta_{G}$ which lead to the regular form. Lastly, a nonlinear gain is given in order to reduce the chattering phenomenon as the state converges asymptotically to the origin.

\section{References}

[1] Drazenovic, B. (1969). "The Invariance Conditions in Variable Structure Systems", Automatica, 5(3), 287-295.

[2] El-Ghezawi, O. M. E., Zinober, A. S. I. and Billings, S. A. (1983). "Analysis and Design of Variable Structure Systems using a Geometric Approach", Int. J. Control, 38(3), 657-671.

[3] Esfandiari, F. and Khalil, H. K. (1991). "Stability Analysis of a Continuous Implementation of Variable Structure Control", IEEE TAC, 36(5), 616-620.

[4] Fliess, M. and Messager, F. (1992). "Sur la Commande en Régime Glissant", C.R. Académie des Sciences de Paris, t.313, Série I, pp. 951-956.

[5] Guckenheimer, J. and Holmes, P. (1983). "Nonlinear Oscillations, Dynamical Systems and Bifurcations of Vector Fields", Applied Mathematical Sciences 42, Springer-Verlag.

[6] Guldner, J. and Utkin, V., "Stabilization of Non-Holonomic Mobile Robots using Lyapunov Functions for Navigation and Sliding Mode Control", Proceedings of the 33rd Conference on Decision and Control, Lake Buena Vista, FL-December 1994, pp. 2967-2972.

[7] Hahn (1963). "Theory and Application of Liapunov's direct Method", Prentice-Hall inc., Englewood Cliffs, N.Y.

[8] Isidori, A. (1989). "Nonlinear Control Systems: An introduction", 2nd edition, CCES, Springer-Verlag.

[9] Lukyanov, A. G. and Utkin, V. I. (1981). "Methods of Reducing Equations for Dynamic Systems to a Regular Form", Automation and Remote Control, 42(4), $413-420$.

[10] Perruquetti, W., Richard, J. P. and Borne, P. (1996). "Lyapunov Analysis of Sliding Motions: Application to Bounded Control", Mathematical Problems in Engineering, 3, 1-25.

[11] Perruquetti, W., Richard, J. P. and Borne, P. (1997). "A Generalized Regular Form for Sliding Mode Stabilization of MIMO Systems", Proceedings of the 36th Conference on Decision and Control.

[12] Sira-Ramirez, H. (1988). "Differential Geometric Methods in Variable-Structure Control", Int. J. Control, 48(4), 1359-1390.

[13] Sira-Ramirez, H. (1989). "Sliding Regimes in general Non-Linear Systems: a Relative Degree Approach", Int. J. Control, 50(4), 1487-1506.

[14] Slotine, J. J. E. (1984). "Sliding controller design for non-linear systems", Int. J. Control, 40(2), 421-434.

[15] Utkin, V. I. (1992). "Sliding Modes in Control Optimization", CCES, SpringerVerlag. 


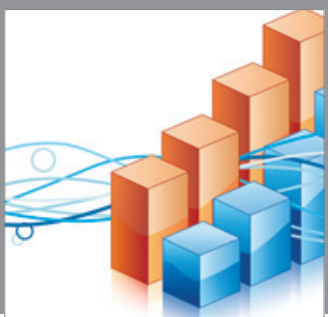

Advances in

Operations Research

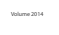

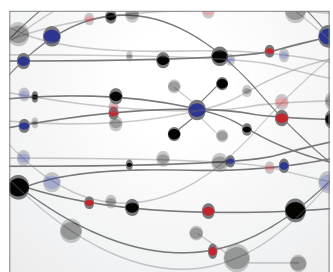

\section{The Scientific} World Journal
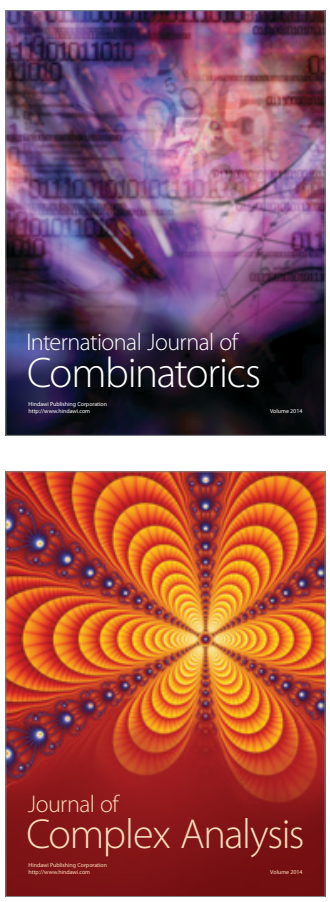

International Journal of

Mathematics and

Mathematical

Sciences
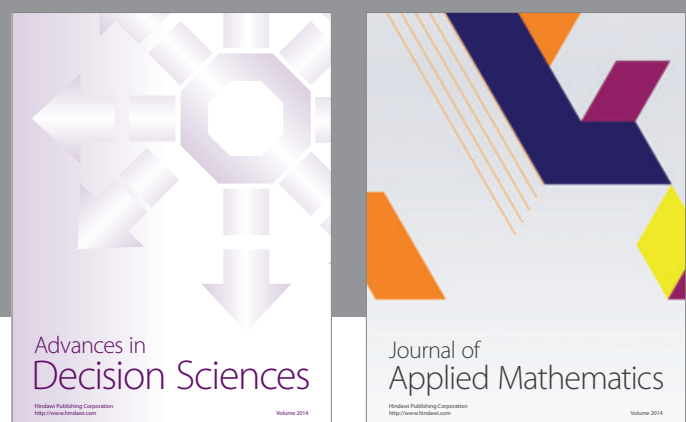

Journal of

Applied Mathematics
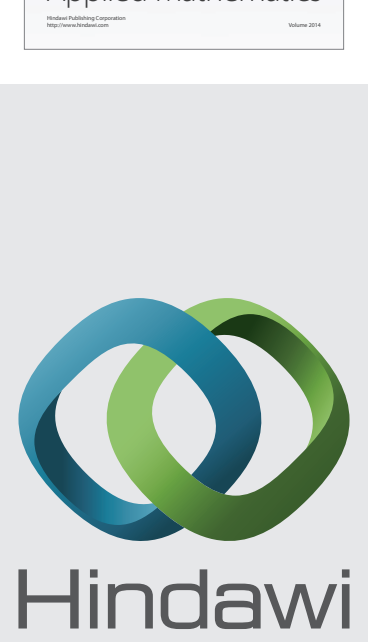

Submit your manuscripts at http://www.hindawi.com
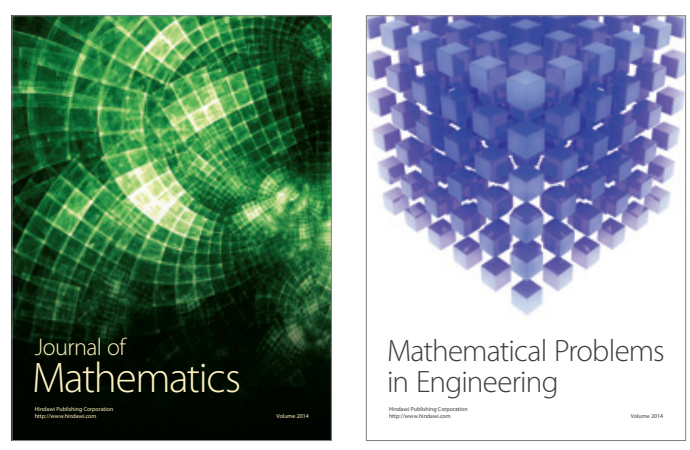

Mathematical Problems in Engineering
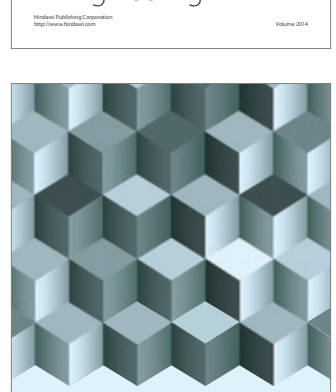

Journal of

Function Spaces
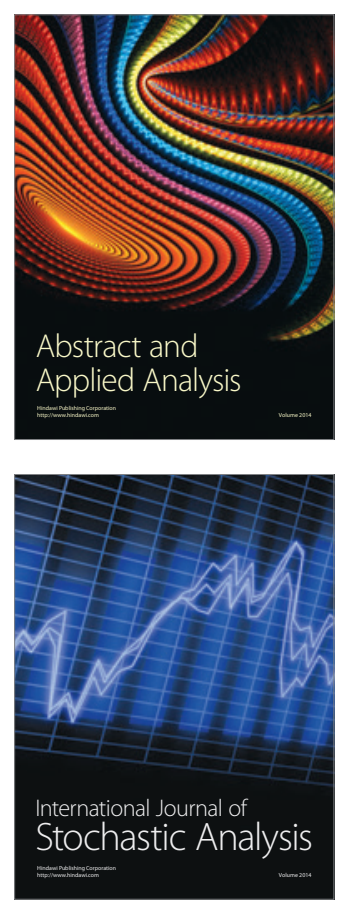

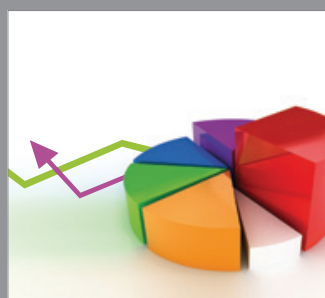

ournal of

Probability and Statistics

Promensencen
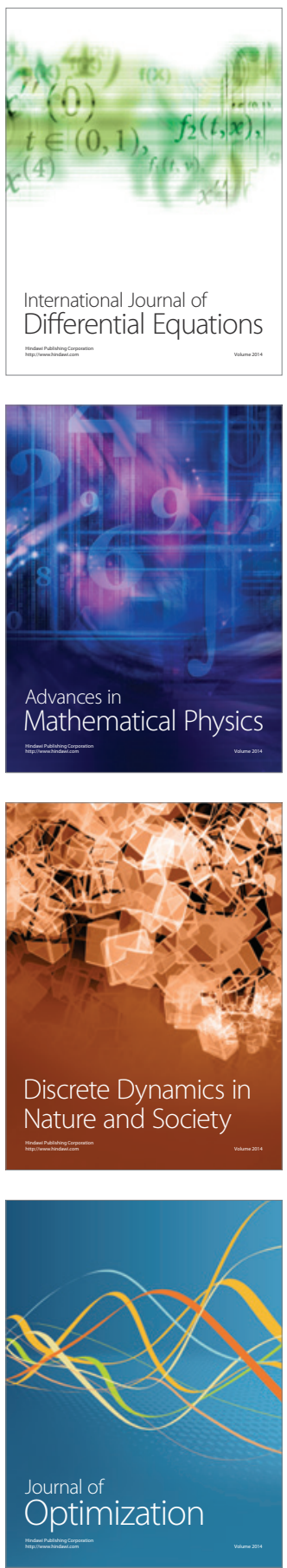